\title{
Tool for supporting local energy strategies: forecasting energy plans with Artificial Neural Network in Umbria Region
}

\author{
Domenico Palladino ${ }^{1, *}$ and Iole Nardi ${ }^{1}$ \\ ${ }^{1}$ Italian National Agency for New Technologies, Energy and Sustainable Economic Development \\ (ENEA), DUEE Department, 00123 via Anguillarese 301 Santa Maria di Galeria (Rome), Italy
}

\begin{abstract}
In order to reduce the greenhouse gas emission and to improve the energy efficiency of buildings, European Member States have to plan medium-to-long term strategies as reliable as possible. In this context, the present work aims to discuss the potentiality of Artificial Neural Network (ANN) as a support tool for medium-to-long term forecasting analysis of energy efficiency strategies in Umbria Region (central Italy) chosen as case study. Parametric energy simulations of several archetypes buildings were carried out in compliance with the current Italian regulations by changing the form, thermal properties, boundary conditions, and technical building systems. An ANN able to forecast primary energy need was trained to forecast the energy need of building-stock of Umbria Region and to evaluate the effectiveness of several potential energy actions (such as thermal coat or technical building systems replacement) over the years. Results confirm the potential of use of ANN as a support tool in energy forecasting analysis for local Authorities. ANN is capable of forecasting different future scenarios allowing correctly planning energy actions to be implemented as well as their priority. The results open to several scenarios of interest, such as the application of the same approach at national level.
\end{abstract}

\section{Nomenclature}

\begin{tabular}{|c|c|}
\hline ANN: & Artificial Neural Network (-) \\
\hline BSU & Building Stock of Umbria Region \\
\hline BU & building unit (apartment of building) \\
\hline Current & related at the current state of Buildings \\
\hline EES & Energy Efficiency Strategy \\
\hline EP & Primary Energy \\
\hline ES & Energy Strategy \\
\hline glo & global \\
\hline HDD & Heating Degree Days \\
\hline 1 & Indicator \\
\hline IBS & Italian Building Stock \\
\hline
\end{tabular}

\footnotetext{
* Corresponding author: domenico.palladino@enea.it
} 


$\begin{array}{ll}\text { MLP } & \text { Multi-Layer Perceptron network } \\ \text { MSE } & \text { Mean Squared Error } \\ \text { R } & \text { Regression } \\ \text { RR } & \text { renovation rate } \\ \text { TBS } & \text { Technical Buildings System }\end{array}$

\section{Introduction}

Due to the human and other natural factors, $\mathrm{CO}_{2}$ emissions are significantly raised leading to an increase of global average temperatures. The climate change entails alarming consequences such as desertification, rising and souring of the oceans, and more frequent extreme atmospheric phenomena. The $\mathrm{CO}_{2}$ emission reduction plays a key role in the current energy policies where sustainable solutions and energy actions are promoted, leading to the green economy transition.

The adoption of focused energy strategies is an unavoidable challenge of all European Countries with the common goal of reducing fossil fuels and of increasing the energy efficiency. In this framework, it is relevant to identify the most energy-intensive sectors in order to adopt appropriate energy strategies.

According to annual national report [1], the energy need of building sector is increased of $0.6 \%$ in 2019 remaining the most energy-intensive sector with about $40 \%$ of total energy consumptions. In order to reduce the $\mathrm{CO}_{2}$ emissions and to improve buildings' energy efficiency, different national energy strategies are undertaken, such as tax incentives or deduction with the aim of reducing total energy need of building sector of 25.5 Mtep by 2020 . These energy strategies allowed an energy saving of 17.6 Mtep in 2014-2019 period [1] near the main goal but still lower than expectations.

These actions are medium-to-long term energy strategies, whose energy saving calculation are based on forecasting energy models, whose effectiveness can significantly affect the energy scenarios. Statistical models are the most common tools adopted for this kind of analysis; however, regression analysis, fuzzy systems, and Artificial Neural Networks (ANN) are also interesting tools proposed in the Literature [2-7]. In particular, ANNs are applied in different fields with several purposes [8]: for electricity consumption forecasting of buildings $[6,7]$ or for building energy evaluation in agreement with national regulations $[9,10]$. Some of these studies highlighted the potential of ANNs in testing the effectiveness of medium-to-long term energy solutions in emissions reductions, energy saving, and energy consumptions [11-13] for specific contexts (such as Beijing [11] and Europe area $[12,13]$ ) but taking into account different sectors (civil sector, agriculture, industry, construction industry, transportation and service sector).

In this framework, the proposed paper aims to show the potential of Artificial Neural Network application in forecasting energy analysis, in order to identify the best energy strategies and energy efficiency improvement on building sector only, representing a new and specific application of ANN. The Umbria Region (central Italy) and its building stock was chosen as territory case study.

The paper aims at assessing the advantage of use of ANN as a support tool for energy strategy decision for local authorities, increasing the energy strategy impact by:

a. choosing the buildings to be renovated;

b. identifying the best energy solution to be applied for each building.

The manuscript is structured as follows: section 2 describes the methodology procedure employed for developing and training Artificial Neural Network (section 2.1) and the evaluation of the current energy state of building sector in Umbria Region (section 2.2). 
Results related to energy forecasting with ANN are discussed in section 3, whilst the main conclusions are exposed in section 4 .

\section{Materials and Methods}

The aim of the paper is to develop a new algorithm capable of forecasting the energy need of building over the years by taking into account different possible energy scenarios adopted by local authorities. The new algorithm should be able to:

a. assess the current energy need of each building unit (BU) and of the whole building stock of Umbria Region (BSU);

b. forecast the energy need over the years of each BU and of the whole BSU;

c. help in enhancing the energy strategies in order to optimizing the energy saving.

The research procedure elaborated in this work, shown in Fig. 1, concerns the following key points:

1. Analysis of the Italian building stock (IBS): energy analysis of the current state of BS, which is the starting point of the forecasting studies. A deep analysis of IBS, with a focus on the Umbria Region, was carried out for defining the main features and thermal characteristics of buildings (archetypes definition);

2. Current energy status of building stock of Umbria Region: based on results from the previous step, the Global Primary Energy need ( $\left.\mathrm{EP}_{\text {glo }}\right)$ was estimated according to UNI TS $11300-1$ [14], and it was considered as the current energy status of BSU (EP glo-Current). Besides, based on national reports [1] and [15], the most incentivized solutions (such as replacement of windows or of existing boiler with more efficient ones) were chosen as possible energy efficiency strategies (EES) to be applied;

3. Artificial Neural Network implementation: based on step 1, several archetypes were defined which were used for carrying out parametric energy analysis in compliance with [14]. Different climatic weather conditions, thermal properties of building envelope, and technical building systems (TBS) were taken into account and more than 10 million of energy simulations were performed. Based on energy simulations, an ANN was developed by appropriately choosing the input and target data. To check the goodness of the training process the Regression values (R) and the Mean Squared Error (MSE) were used as control parameters;

4. Validation of Artificial Neural Network: downstream of the ANN training process, the current energy need of BSU was also assessed with ANN (EP glo-ANN-Current) and it was compared with EP glo-Current in order to check the reliability of the ANN itself;

5. $1^{\text {st }}$ Energy Forecasting: once validated, the developed ANN was used to predict the energy need of BSU for each considered EES (EP glo-ANN-ES1) over the years (up to the next 25 years);

6. Improvements of energy savings for each EES (IEES): based on ANN predictions several improvements were identified in order to maximize the energy savings;

7. $2^{\text {nd }}$ Energy Forecasting: ANN was thus used one more time to assess the energy need of BSU for each IEES ( EP $_{\text {glo-ANN-ES2 }}$ and $\mathrm{EP}_{\text {glo-ANN-ES3 }}$ ) over the years (up to 25 years later);

8. Energy scenarios comparison: finally, all the energy forecasts were compared to each other. 


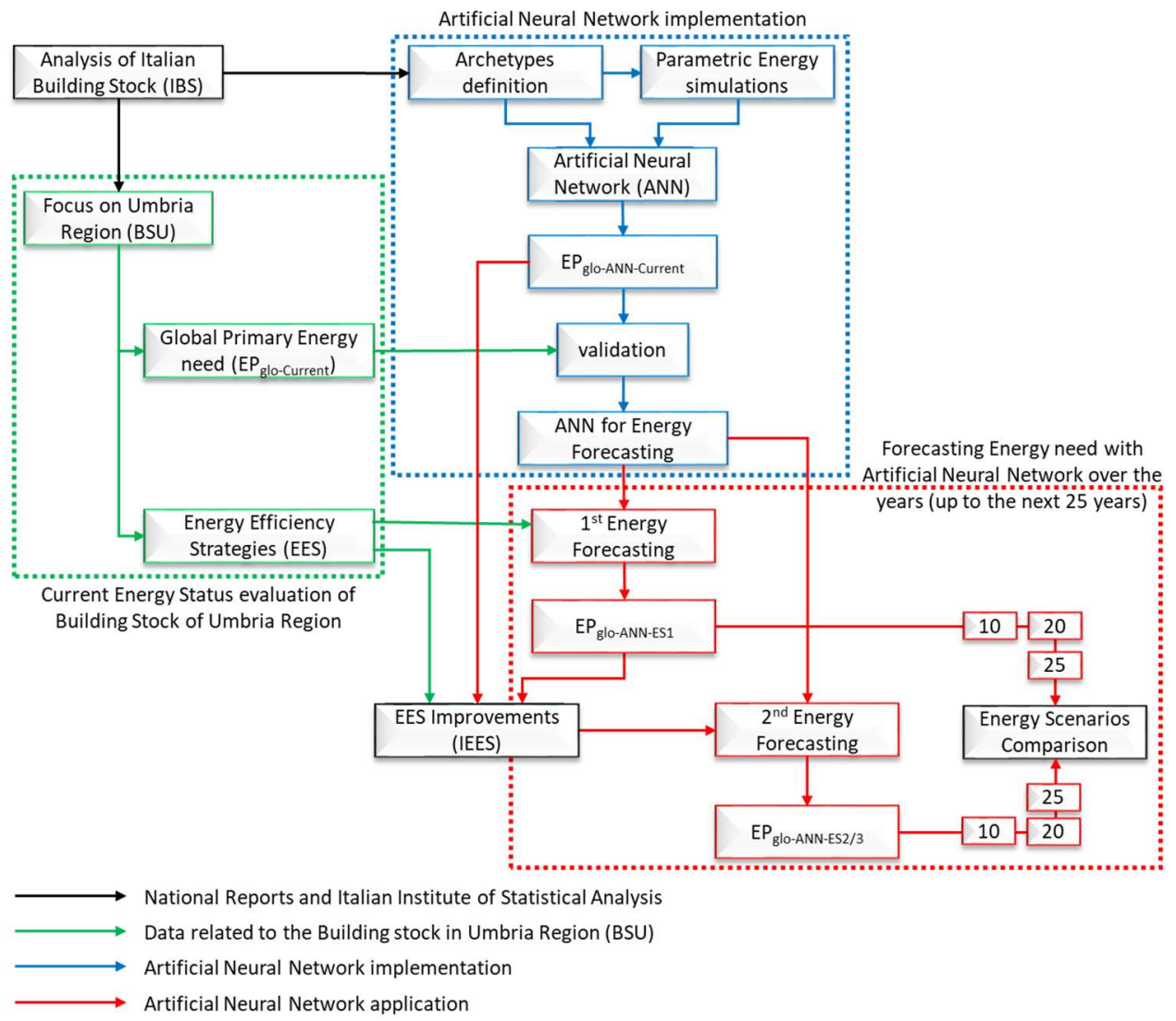

Fig. 1. Research procedure adopted for the development of Artificial Neural Network able to forecast global primary energy need of buildings.

\subsection{Artificial Neural Network: training process}

According to the purpose of the paper, the Artificial Neural Network has to be capable of forecasting the global primary energy need of buildings in agreement with the current national regulations. Parametric energy simulations were carried out in compliance with [14] of several archetypes expressly defined on available data of Italian Institute of Statistical Analysis (ISTAT) [16] which are representative of the whole IBS in terms of type, dimensions, and thermal properties.

A parametric analysis was performed by taking into account different values of the following parameters:

1. architectural and dimensional characteristics of BU (Table 1);

2. thermal properties of building envelope (Table 2);

3. thermal transmittance of windows (values in $1.3-3.0 \mathrm{~W} / \mathrm{m}^{2} \mathrm{~K}$ range were assumed);

4. climatic weather conditions (25 locations were chosen with different Heating Degree Day ranging from 568 to 4264 HDD);

5. boundary conditions of each BU (Table 1);

6. TBS for space heating, domestic hot water production, and cooling (such as electric boiler, standard boiler, condensing boiler, and heat pump); 
7. integration of TBS with renewable energy sources (such as thermal solar collectors and photovoltaic systems).

Data related to weather conditions (global solar radiation and Heating Degree Days), geometry (net surface, net volume, building envelope surfaces), thermal properties (thermal transmittance), and TBS (type of generation system, power, nominal efficiency, and mainly energy carrier) were provided as input parameters in the ANN training process, whilst the primary energy need $\left(\mathrm{EP}_{\mathrm{glo}}\right)$ was supplied as target.

Table 1. Values of building typology, boundary conditions, and dimensional characteristics of archetypes set for the parametric analysis.

\begin{tabular}{c|clcc} 
Archetypes & $\begin{array}{c}\text { Building typology } \\
{[-]}\end{array}$ & $\begin{array}{l}\text { Boundary conditions } \\
{[-]}\end{array}$ & $\begin{array}{c}\text { Net surface } \\
{\left[\mathbf{m}^{\mathbf{2}}\right]}\end{array}$ & $\begin{array}{c}\text { Window surface } \\
{\left[\mathbf{m}^{\mathbf{2}}\right]}\end{array}$ \\
\hline 1 & & single floor & 100 & 13.7 \\
2 & Single-family & single floor & 135 & 18.0 \\
3 & & single floor & 168 & 24.5 \\
\cline { 2 - 5 } 4 & & ground floor & 80 & 11.0 \\
5 & & intermediate floor & 100 & 13.7 \\
6 & & top floor & 120 & 17.5 \\
7 & & ground floor & 80 & 11.0 \\
8 & \multirow{2}{*}{ Multifamily } & intermediate floor & 100 & 13.7 \\
9 & & top floor & 120 & 17.5 \\
10 & & ground floor & 80 & 11.0 \\
11 & & intermediate floor & 100 & 13.7 \\
12 & & top floor & 120 & 17.5
\end{tabular}

Table 2. Values of the thermal properties of building envelope set for the parametric analysis.

\begin{tabular}{c|cccc}
$\begin{array}{c}\text { Building } \\
\text { envelope }\end{array}$ & Typology & $\begin{array}{c}\text { Thermal } \\
\text { insulation }[\mathbf{c m}]\end{array}$ & $\begin{array}{c}\text { Thickness } \\
{[\mathbf{c m}]}\end{array}$ & $\begin{array}{c}\text { Thermal } \\
\text { transmittance } \\
{[\mathbf{W} / \mathbf{m} 2 \mathbf{K}]}\end{array}$ \\
\hline \multirow{5}{*}{ Walls } & masonry & 0 & $24-64$ & $3.22-0.95$ \\
& beams and pillars & 0 & $19-64$ & $1.56-0.60$ \\
& masonry & 5 & $29-69$ & $0.53-0.38$ \\
& beams and pillars & 5 & $24-69$ & $0.49-0.33$ \\
& masonry & 10 & $34-74$ & $0.29-0.24$ \\
& beams and pillars & 10 & $29-74$ & $0.27-0.19$ \\
& New construction & $>10$ & $30-58$ & $0.21-0.13$ \\
\hline \multirow{3}{*}{ Pavements } & - & 0 & $34-56$ & $1.68-1.45$ \\
& - & 5 & $39-61$ & $0.67-0.55$ \\
& - & 10 & $44-66$ & $0.38-0.34$ \\
& - & $>10$ & $48-72$ & $0.27-0.20$ \\
\hline \multirow{3}{*}{ Ceiling } & - & 0 & $8-36$ & $1.68-1.45$ \\
& - & 5 & $13-41$ & $0.67-0.55$ \\
& - & 10 & $18-54$ & $0.38-0.34$ \\
& - & $>10$ & $22-64$ & $0.27-0.20$
\end{tabular}

The network was implemented by adopting the most common architecture of ANN, i.e. a multi-layer feed forward neural network (MLP), consisting of one input layer, one hidden layer and one output layer. In this pattern, a sigmoidal function was chosen as transfer function for both the hidden layer and output one, allowing to simplify the calculation of 
gradient of error and reducing the computational time required for the training of network. The training process was performed by using the Back Error Propagation algorithm, obtaining a reliable ANN by using 13 neurons in the hidden layer. The effectiveness of the training process was checked with Regression values (higher than 0.998 in training, validation, test, and global processes) and by the mean error (Fig. 2) calculated as the difference between the target and the output of ANN. As can be noticed, the mean error is mainly in $-0.02+0.02$ range (more than $98 \%$ of cases) to which corresponds MSE values equal to $4.228 \cdot 10^{\wedge-5}, 4.179 \cdot 10^{\wedge-5}, 4.180 \cdot 10^{\wedge-5}$ for the training, validation, and testing processes respectively.

The more probable mean error returned by ANN (Fig. 2) falls in a very small and acceptable range; therefore, taking also into account the Regression and MSE values obtained for all the development processes, a good reliability of ANN can be confirmed.

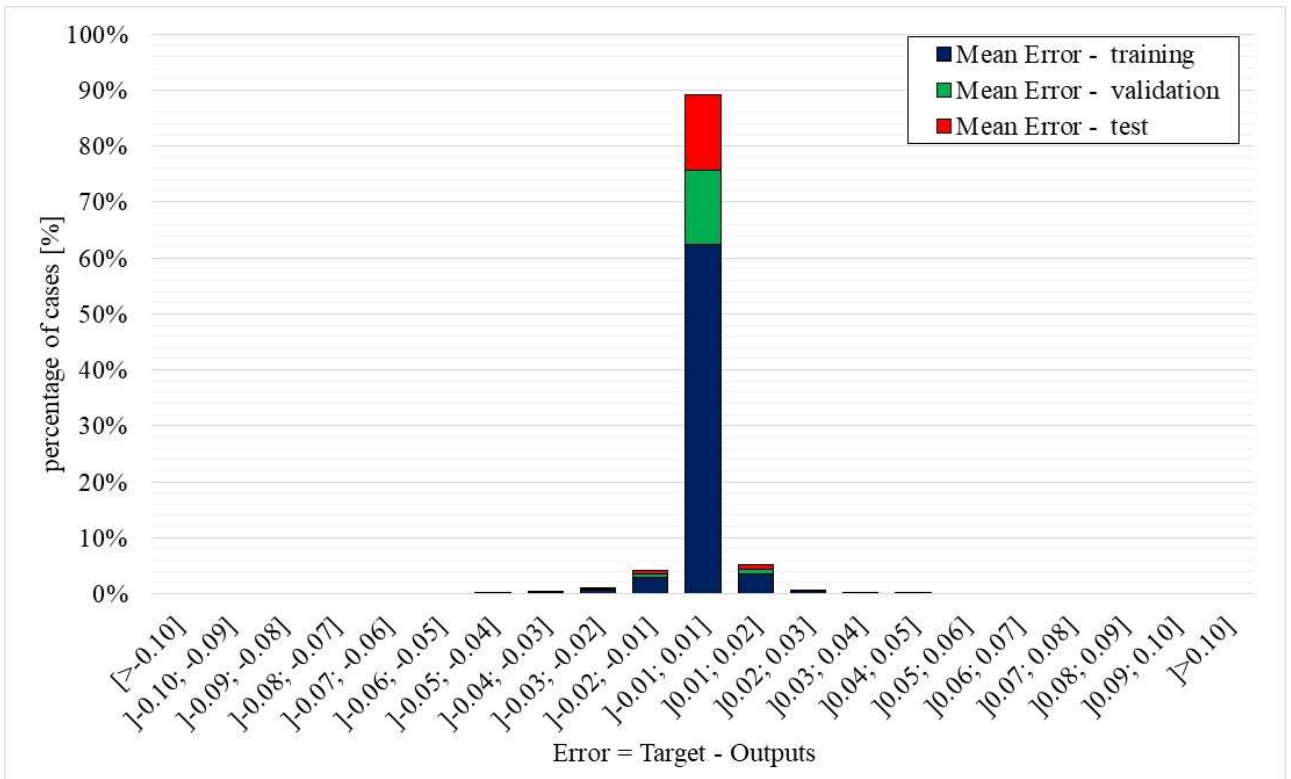

Fig. 2. Mean Error returned by development processes of ANN.

\subsection{Energy consumption of building stock of Umbria Region}

As shown in Fig. 1, one of the most important point to be discussed is the current energy

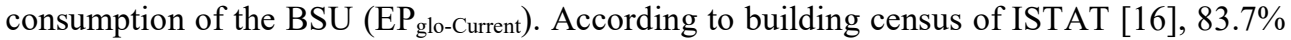
of buildings (about 167200) are residential buildings which have poor energy performance, being built before 1990 (more than 90\% of whole residential buildings in Umbria Region). The typical net surface of residential buildings is in $60-150 \mathrm{~m}^{2}$ range, mainly heated with independent heating system (more than 66\%). Electric energy and natural gas are the energy carriers mainly used.

Basing on the state of the art of BSU, the same archetypes used for the parametric simulations (which mainly represent the national heritage with good approximation) were employed for simulating the $\mathrm{EP}_{\text {glo-Current }}$ in compliance with [14] considering the real thermal characteristics and number of buildings in each municipality of Umbria. At the same time, the same data of the archetypes used for $\mathrm{EP}_{\text {glo-Current }}$ calculation were also supplied to the ANN for the EP glo-ANN-Current evaluation.

EP glo-ANN-Current, expressed in tons of oil equivalent (toe) and in $\mathrm{kWh} / \mathrm{m}^{2}$, was thus estimated and compared to $\mathrm{EP}_{\text {glo-Current }}$ allowing a twofold purpose: 
1. to define the $\mathrm{EP}_{\text {glo-Current }}$ which represents the primary energy need required for heating, cooling, and domestic hot water production of BSU;

2. to check the reliability of the ANN.

Results of this comparison are shown in Fig. 3 for the main representative municipality of Umbria (the ones with the higher population density): the shade of blue bars are the mean $\mathrm{EP}_{\text {glo-ANN-Current }}$ whilst the shade of red bars are the $\mathrm{EP}_{\text {glo-Current }}$ (resulting from energy simulations). $\mathrm{EP}_{\text {glo }}$ was calculated for three built period as the mean values of all BSU: before 1980, between 1981-2000, and after 2001.

According to Fig. 3, the difference between $\mathrm{EP}_{\text {glo-ANN-Current }}$ and $\mathrm{EP}_{\text {glo-Current }}$ is about \pm 8.5 $\mathrm{kWh} / \mathrm{m}^{2}$, which has been considered acceptable for the purpose of the work.

Based on the energy simulations of BSU, the total Primary Energy of all the residential units of the Region was also estimated (considering the real number of building units, their thermal characteristics, and the energy need simulated by ANN), obtaining an energy need of 869,110 toe, corresponding to a mean $\mathrm{EP}_{\text {glo }}$ of $206 \mathrm{kWh} / \mathrm{m}^{2}$. These values were assumed as the starting point of energy forecasting analysis.

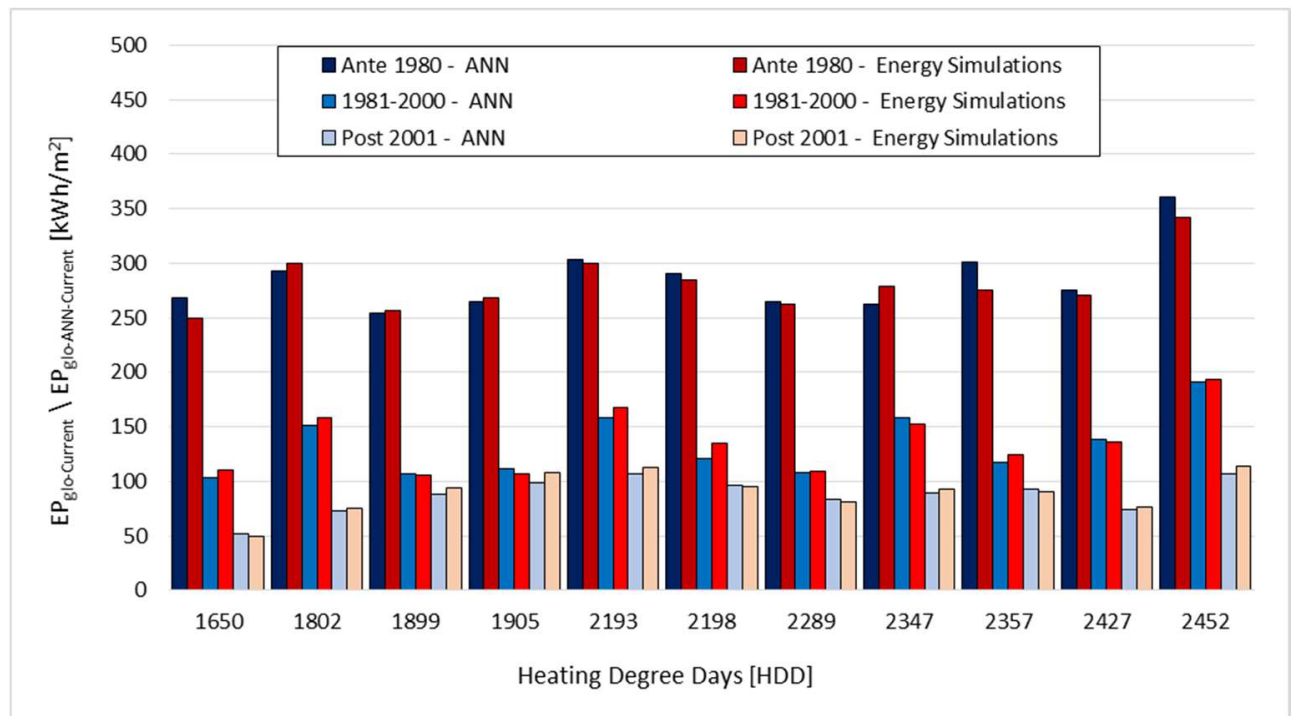

Fig. 3. Primary energy need comparison: $A N N$ (EP $\left.{ }_{\text {glo-ANN-Current }}\right)$ vs. energy simulations ( $\left.\mathrm{EP}_{\text {glo-Current }}\right)$ in the most representative municipality of Umbria Region (the ones with the higher population density).

\section{Results and Discussion}

Once defined the current energy status of the BSU (starting point), the developed ANN was therefore used for energy forecasting, by adopting six different EES (which refer to the energy efficiency measures to be employed) and three different energy strategies (ES which refer to the criteria for selecting the renovation rate).

The energy efficiency scenarios are:

- EES1: replacement of existing windows with most efficient ones;

- EES2: replacement of existing boiler with condensing boiler;

- EES3: replacement of existing boiler with hydronic heat pump;

- EES4: deep renovation of building envelope (thermal coat installation and simultaneous windows replacement); 
- EES5: deep renovation of building (thermal coat installation and simultaneous windows replacement) plus the replacement of existing boiler with condensing boiler;

- EES6: deep renovation of building (thermal coat installation and simultaneously windows replacement) plus the replacement of existing boiler with heat pump.

Energy forecasting up to 25 years was performed with ANN by adopting a specific renovation rate (Table 3), for each scenario, chosen in agreement with local and national reports $[15,17]$. The energy forecasting was carried out for the following energy strategies depending on each EES and on the adopted improvements (IEES):

1. ES1: local and national reports $[15,17]$ provide different information of the buildings that were renovated until 2019-2020 such as the year of construction, dimensions, and locations. Therefore, according to [15, 17], buildings with the same characteristics were taken into account and chosen in ES1 as to be renovated. Results of this scenario were EP glo-ANN-ES1;

2. ES2: basing on $\mathrm{EP}_{\text {glo-ANN-Current }}$ and $\mathrm{EP}_{\text {glo-ANN-ES1, buildings with higher energy }}$ consumptions were chosen as the ones to be renovated, whilst the same locations adopted in ES1 were kept. Results of this scenario were $\mathrm{EP}_{\text {glo-ANN-ES2; }}$;

3. ES3: basing on EP glo-ANN-Current and EP glo-ANN-ES1 (outcomes of ANN), buildings with higher energy consumptions and located in the most energy-intensive municipality of Umbria Region were chosen as the ones to be renovated. Results of this scenario were EP glo-ANN-ES3.

Results of energy forecasting with ANN up to 25 years are shown in Fig. 4 for each EES and for all the adopted ES (black squares and black trend line for ES1, in red and blue results for ES2 and ES3 respectively).

Basing on results of Fig. 4, new indicator was introduced ( $\mathrm{I}_{\mathrm{EES}-\mathrm{RR}}$ ) to analyse and compare each other the EESs: it was calculated as the ratio between the energy saving predicted for each EES and the adopted renovation rate (Table 3), and it represents the weight that each EES has on energy saving. Therefore, the lower the IEES-RR, the lower the energy benefit.

Based on IEsS-RR and on Fig. 4, it is possible, for each ES, to state the following:

- ES1: the best solutions for ES1 are the two deep renovations of buildings (EES4, EES5, and EES6), which allow reducing the total primary energy of about 1.1$1.3 \%$ with an IEES-RR between 16.1-18.9\%. The EES3 leads an energy saving of $3.4 \%$ next to 25 years (I reduction of $1.7 \%$ with $\mathrm{I}_{\text {EES-RR }}$ equal to $3.0 \%$. Although EES2 is the most widespread solution, it allows obtaining an energy saving of $1.6 \%$ (at $25^{\text {th }}$ year) with an IEES-RR of only $0.75 \%$;

- ES2: with the support of ANN in energy strategy improvements, a higher energy saving of 2-13\% was obtained for all the EESs. The best solutions (EES4, EES5, and EES6) allow reducing the total primary energy of about $1.3-1.5 \%$ with an $\mathrm{I}_{\text {EES-RR }}$ between 18.6-21.2\%. The EES3 leads an energy saving of 3.8\% (IEES-RR equal to $7.8 \%$ ), whilst EES1 of $1.8 \%$ with $\mathrm{I}_{\mathrm{EES}-\mathrm{RR}}$ equal to $3.1 \%$. In this case, EES2 allows obtaining an energy saving of $1.8 \%$ with an $\mathrm{I}_{\text {EES-RR }}$ of only $0.82 \%$;

- ES3: this improved strategy allows increasing the energy saving from $18 \%$ to $34 \%$ with respect ES1. The best solutions remain EES4, EES5, and EES6 with an energy saving of about $1.4-1.6 \%$ with an IEES-RR between $20.6-23.2 \%$. The EES3 leads an energy saving of $4.1 \%$ ( ( $\mathrm{IEES}_{\mathrm{E}-\mathrm{RR}}$ equal to $8.5 \%$ ), whilst EES1 allows an energy reduction of $2.0 \%$ with $\mathrm{I}_{\text {EES-RR }}$ equal to $3.6 \%$. The worse solution is still the EES2 with an energy saving of $2.2 \%$ but with an $\mathrm{I}_{\mathrm{EES}-\mathrm{RR}}$ of only $1.0 \%$. 
Table 3. Renovation rate of each energy scenario according to $[15,17]$.

\begin{tabular}{c|c} 
EES & Renovation rate in Umbria Region [\%] \\
\hline 1 & 0.57 \\
2 & 2.20 \\
3 & 0.48 \\
4 & 0.07 \\
5 & 0.07 \\
6 & 0.07
\end{tabular}
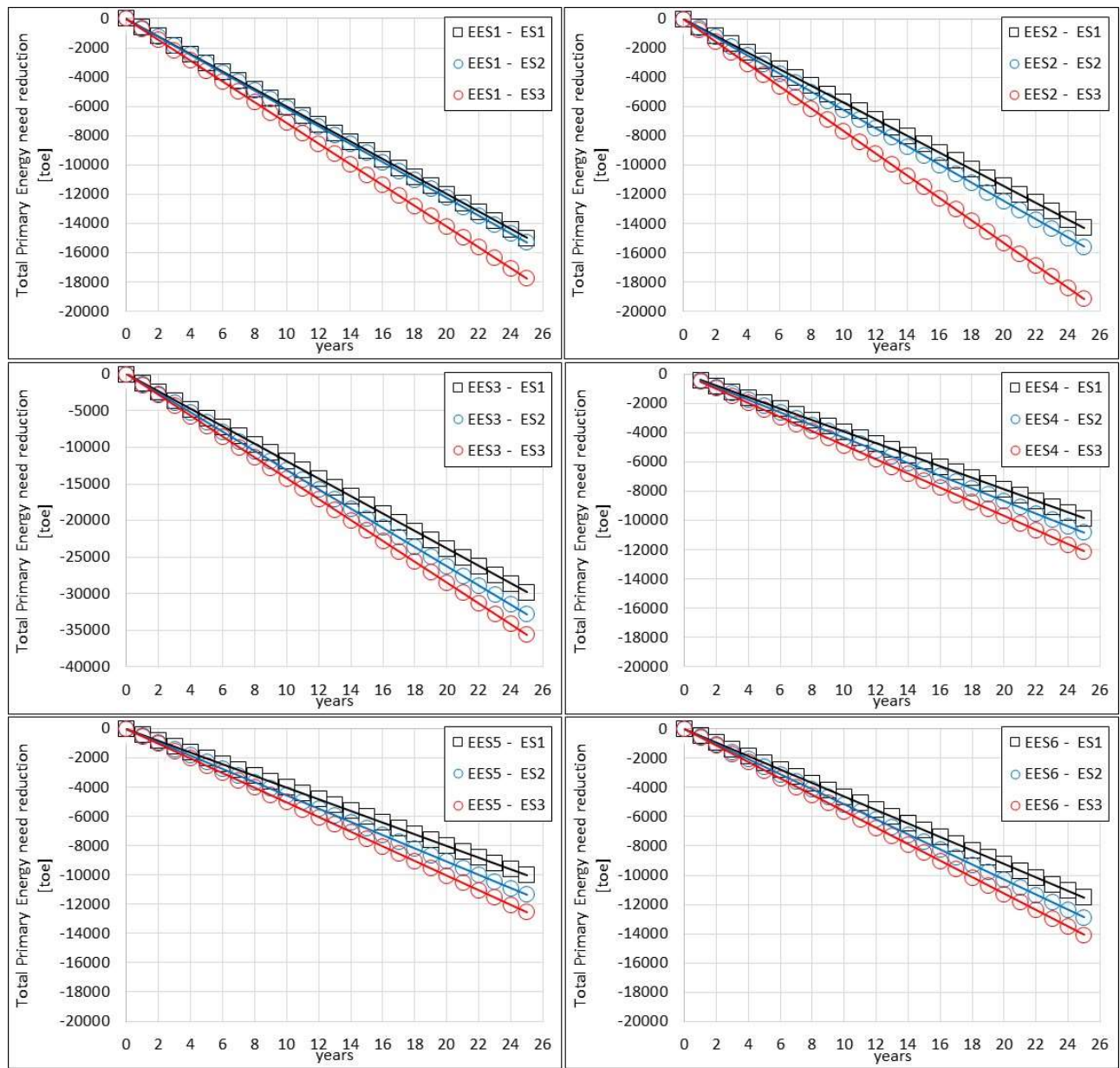

Fig. 4. Energy strategies and energy scenarios comparison.

According to these results, the best solutions seem to be EES4, EES5, and EES6, which are the ones with the higher $\mathrm{I}_{\mathrm{ESS}-\mathrm{R} R}$, i.e. allow obtaining a higher energy savings if the same renovation rate is adopted. On the other hand, EES2 is the energy solution with the higher energy saving but mainly due to the higher renovation rate (equal to $2.2 \%$ ); i.e. although it is a very widespread solution, individually, it does not lead significant energy saving.

Altogether, in the ES1 all the proposed EES strategy lead to an energy saving of about 90,400 toe, equal to an energy reduction of $10.4 \%$ in 25 years. With the support of ANN, it is possible to increase the energy saving of about $9 \%$ (ES2) and $23 \%$ (ES3) compared to ES1, corresponding to an energy reduction of $11.3 \%$ (ES2) and of $12.8 \%$ (ES3) in 25 years. 
According to these results, ANN seems to be a very useful and powerful support tool for energy forecasting analysis capable of significantly increasing the achievable energy savings. However, more accurate analyses are needed, for instance by taking also into account economic aspect in order to have a more exhaustive overview of each EES.

\section{Conclusions}

The aim of this work is to show the potential of Artificial Neural Network (ANN) in energy forecasting analysis in order to increase the energy saving of energy efficiency actions in the building sector.

To achieve this goal, the Umbria Region (central Italy) was chosen as case study. Once defined the current energy status of the building stock of Umbria, and trained an ANN in agreement with the current national energy regulations, several energy solutions are defined to improve the energy efficiency of residential buildings.

The accuracy and effectiveness of ANN use in energy forecasting analysis was confirmed by the results. Particularly, the ANN can be a useful and powerful tool not only for energy prediction of the whole building stock, but also for energy strategy improvement. In fact, ANN allows optimizing the energy saving of each energy solution adopted to improve the energy efficiency of buildings. With respect the standard energy strategy defined in compliance with local and national reports, the improved energy strategies with the support of ANN allow reaching an increasing energy saving up to 34\% (considering the replacement of existing boiler with condensing boiler). ANN allows increasing the energy saving of about $9-23 \%$, corresponding to a total energy reduction of $11-13 \%$ in 25 years.

The present work is only a preliminary study that highlights the potential of ANN in energy forecasting analysis, but further and more accurate analysis are ongoing by also taking into account the economic aspect in order to have a more exhaustive overview. However, it opens to future scenarios of considerable interest as well as to new opportunities for national and local authorities in energy strategy definition and optimization.

\section{References}

1. Ministry of Economic Development, National Energy Situation in 2019 (2020)

2. L. Zhang and J. Wen, Energy Build, 244, 111026 (2021)

3. S. Aslam, H. Herodotou, S. M. Mohsin, N. Javaid, N. Ashraf, and S. Aslam, Energy Reviews, 144, 110992 (2021)

4. A. Mashlakov, T. Kuronen, L. Lensu, A. Kaarna, and S. Honkapuro, Appl. Energy, 285, 116405 (2021)

5. C. A. Severiano, P. C. de L. e. Silva, M. Weiss Cohen, and F. G. Guimarães, Energy, 171, 764-783 (2021)

6. S. Javeed Nizami and A. Z. Al-Garni, Energy Policy, 23, 1097-1104 (1995)

7. A.S. Ahmad, M.Y. Hassan, M.P. Abdullah, H.A. Rahman, F. Hussin, H. Abdullah, R. Saidur, Renewable and Sustainable Energy Reviews, 33, 102-109 (2014)

8. Nova publishers. Artificial Neural Networks New Research: Applications of artificial neural networks to energy and buildings (2017)

9. C. Buratti, M. Barbanera, and D. Palladino, Appl. Energy, 120, 125-132 (2014)

10. C. Buratti, F.C. Orestano, and D. Palladino, Energy Procedia, 101, 176-183 (2016)

11. D. Zhang, G. Liu, C. Chen, Y. Zhang, Y. Hao, and M. Casazza, Energy Policy, 127, 350-360 (2019) 
12. B. D. Gvozdenac Urošević and D. J. Đozić, Energy, 227, 120470 (2021)

13. D. J. Đozić and B. D. Gvozdenac Urošević, Energy, 174, 488-496 (2019)

14. UNI TS 11300 - 1 Energy Performance of Buildings - Evaluation of energy need for space heating and cooling (2014) (in Italian)

15. Umbria Region. Regional Environmental and Energy Strategy 2014-2020 (2020)

16. Italian National Institute of Statistics, available online: http://dati.istat.it/

17. ENEA, Annual report on tax deduction (2020) 